\title{
Assessment of efficacy of IV $\omega$-3 PUFA supplementation in patients of predicted severe acute pancreatitis using the positive predictive value of Balthazar score within 48 hours of onset
}

\author{
Rao S. ${ }^{1}$, Kunte $\mathbf{A}^{2}$ \\ ${ }^{1}$ Professor Shilpa Rao, Professor and Unit Head, Department of General Surgery, Seth G. S. Medical College and K.E.M. \\ Hospital, Mumbai, India. ${ }^{2}$ Dr. Aditya Kunte, Speciality Medical Officer, Department of General Surgery, Seth G.S. \\ Medical College and K.E.M. Hospital, Mumbai, India.
}

Address for correspondence: Professor Shilpa A. Rao, Department of Surgery, Seth G. S. Medical College \& KEM Hospital, Acharya Donde Marg, Parel, Mumbai, Maharashtra. E-mail address: ateeshilpa@gmail.com

\begin{abstract}
Introduction: Since $50 \%$ of the mortalities in acute pancreatitis (AP) occur within the first week, early prediction of its outcomes is vital. The CT severity index (CTSI) reliably predicts outcomes when calculated 4-7 days after onset. However, a therapeutic window for early aggressive intervention is lost. The Balthazar score can be calculated within 48 hours of onset, thus being a diagnostic as well as a prognostic investigation. Omega-3 polyunsaturated fatty acids $(\omega-3$ PUFA) have a beneficial immunomodulatory effect and improve outcomes in AP. Materials and Methods: 80 patients of clinically predicted severe acute pancreatitis were included. Treatment group received IV $\omega$-3 PUFA supplementation. Balthazar scores were calculated for all patients within 48 hours of onset. Positive predictive value (PPV) for progression to severe acute pancreatitis (SAP) was calculated from the control group. The number of patients predicted to progress to SAP was compared those actually progressing to SAP in the $\omega-3$ PUFA group. Results: PPV for Balthazar score of D and above for predicting progression to SAP was $87.5 \%$ in the control group. $35(87.5 \%)$ patients were predicted to progress to SAP in the $\omega-3$ PUFA group, but only $28(70 \%)$ patients progressed to SAP ( $p=0.0315$ ). Conclusion: Balthazar grade of D and above on a CT scan done within 48 hours of onset of acute pancreatitis can accurately predict the progression of clinically suspected severe acute pancreatitis to severe acute pancreatitis. IV $\omega-3$ PUFA supplementation early in the course of acute pancreatitis significantly improves early clinical outcomes.
\end{abstract}

Key words: Acute pancreatitis, Omega-3 fatty acids, Balthazar score, Immunomodulators, Parenteral nutrition.

\section{Introduction}

The Atlanta guidelines mention an elevated serum lipase or amylase and CT scan as diagnostic modalities for acute pancreatitis. A lipase cut- off of near two- fold above normal has a sensitivity and specificity of $95 \%$ in diagnosing acute pancreatitis $[1,2]$. Markers such as the APACHE-II scores, IL-6, IL-8, IL-10 and CRP which have been used to predict the severity of acute pancreatitis measure the intensity of the systemic inflammatory response syndrome (SIRS) and are not specific to pancreatitis and hence do not contribute to confirming a diagnosis [3-5]. A contrast enhanced CT scan (CECT) is the most accurate single imaging

Manuscript received 20 $0^{\text {th }}$ April 2016

Reviewed: $2^{\text {nd }}$ May 2016

Author Corrected: $14^{\text {th }}$ May 2016

Accepted for Publication $27^{\text {th }}$ May 2016 modality for the diagnosis, staging the severity and detecting the complications of acute pancreatitis [6- 8], with a sensitivity of $87 \%$ and an overall detection rate of over $90 \%$ for pancreatic necrosis $[6,9,10]$. The CT severity index (CTSI) has been shown to reliably predict early and late outcomes of pancreatitis when calculated on a CECT performed 4-7 days after the onset of pancreatitis [11,12]. Since $50 \%$ of the mortalities in pancreatitis occur due to a multi- organ dysfunction syndrome (MODS) within the first week [13], it is essential to be able to predict the outcome of the disease early in its course. The Balthazar score is an objective, reliable, easy method of assessing the severity of pancreatitis, and can hence be calculated from the same CT scan performed to diagnose acute 
pancreatitis [14]. Thus, a Balthazar score within 48 hours of onset of pancreatitis can be used to predict its outcomes and identify the patients with higher risk for local and systemic complications which would necessitate institution of early aggressive therapy.

Fish oil derived omega-3 polyunsaturated fatty acids ( $\omega-3$ PUFA) when administered along with adequate nutritional support has been reported to have a beneficial effect on the immune response; enhancing immunity, reducing inflammatory response, altering cytokine production and hence improving outcomes in AP $[15,16]$.

This study aims to assess the effect of early $\omega$ - 3 PUFA supplementation on clinical outcomes of predicted severe acute pancreatitis (PSAP) by using PPV of Balthazar score on a CT scan done within 48 hours of onset as a marker for clinical outcomes.

\section{Materials and Methods}

Study Design: The study was a single center, openlabel, randomized, controlled, comparative phase IV study to evaluate the immunomodulatory effects of intravenous fish oil derived $\omega$-3 PUFA (Omegaven®Fresenius Kabi, Bad Homburg, Germany) and the related clinical outcomes in 80 consecutive patients with predicted severe acute pancreatitis, by using the positive predictive value of Balthazar score on a CT scan done within 48 hours of onset as a marker for clinical outcomes.

\section{Inclusion Criteria}

1. 18-60 years old patients of either sex, diagnosed with acute pancreatitis. Diagnosis of acute pancreatitis was established by the Atlanta guidelines criteria [17] namely, any two of the following three criteria to be fulfilled:

a. Clinical features suggestive of acute pancreatitis.

b. Serum amylase or lipase levels elevated to more than three times the upper limit of normal.

c. USG or CT showing features of acute pancreatitis.

2. Onset of pain within 24 hours of admission to the hospital.

3. Patients "predicted" to have Severe Acute Pancreatitis (PSAP) by fulfilling the following criteria on admission:

a. Patients showing the signs of a Systemic Inflammatory response syndrome (SIRS), defined by the presence of two or more of the following criteria1: i. Rectal temperature $>38 \mathrm{C}(100.4 \mathrm{~F})$ or $<36 \mathrm{C}(96.8 \mathrm{~F})$

ii. Heart rate $>90$ beats $/ \mathrm{min}$

iii. Respiratory rate $>20 / \mathrm{min}$ or $\mathrm{PaCO} 2<32 \mathrm{mmHg}$.

iv. WBC count $>12000 / \mathrm{mm} 3,<4000 / \mathrm{mm} 3$ or $>10 \%$ bands.

\section{Exclusion Criteria}

1. Patients with known immunodeficient status

2. Primary hypertriglyceridemia

3. On long term cyclo-oxygenase inhibitors (more than 3 months)

4. Severe cardiac disease

5. Known hepatic disorders (total bilirubin $>1.5$ times the upper limit of normal)

6. Psychiatric disorders

7. Known renal compromise (serum creatinine $>2.0 \mathrm{mg} / \mathrm{dl})$.

8. Received parenteral nutrition within 2 weeks of the study.

Study procedure: At the initial screening visit, the diagnosis of acute pancreatitis and presence of SIRS was confirmed on clinical, biochemical (Sr. Lipase, Renal function tests, Liver function tests, $\mathrm{Sr}$. Electrolytes, Complete Hemogram, Arterial Blood Gas analyses) and a contrast enhanced CT scan of the abdomen (CECT). After verifying the absence of any exclusion criteria, such patients, with a predicted severe acute pancreatitis were inducted into the trial.

Body weight measurements were taken of all the patients, and daily caloric requirement was calculated. All patients in the trial received $25 \mathrm{kcal} / \mathrm{kg} /$ day plus $1.5 \mathrm{~g}$ of proteins $/ \mathrm{kg} /$ day of nutrition. Patients were initially started on Total Parenteral Nutrition (TPN). TPN formulation used was Kabiven ${ }^{\mathrm{TM}}$ Peripheral (1400kcal, $1920 \mathrm{~mL}$ emulsion for infusion). The parenteral nutrition was transitioned to enteral nutrition as per dietician's charts as early as possible, keeping the daily nutritional intake constant. Sr. IL-6 levels were sent of all the patients on Day 0. All patients received antibiotic and supportive therapy as per standard protocols for the management of acute pancreatitis.

Patients were then randomly allocated into one of the two treatment arms in a ratio of 1:1. Computer based randomization list was generated and the allocation was done by one of the investigators using sealed envelopes. Both groups were matched for age, sex distribution and levels of inflammatory markers at onset. The treatment arm (Group I) received $\omega-3$ PUFA (Omegaven®, Fresenius Kabi, Bad Homburg, Germany) at the dosage 
of $2 \mathrm{ml} / \mathrm{kg} /$ day i.e., $0.2 \mathrm{~g} / \mathrm{kg} /$ day of $10 \% \omega-3$ PUFA at the rate of $0.05 \mathrm{~g} / \mathrm{kg} / \mathrm{hr}$ i.e., 8 drops per minute for a duration of 5 days.

The patients were assessed at specified intervals for the following parameters: Marshall score [18] done on Day 0 and Day 3 to assess for organ failure. Patients having a Marshall score of 2 or more on day 0 as well as day 3 were diagnosed to have persistent organ failure for > $48 \mathrm{hrs}$, thereby confirming the progression to Severe Acute Pancreatitis. Marshall scores were assessed by the following parameters:

1. $\mathrm{PaO} 2 / \mathrm{FiO} 2$

2. Sr. Creatinine $(\mathrm{mg} / \mathrm{dL})$

3. Systolic blood pressure

4. Arterial $\mathrm{pH}$

Liver function tests, Sr. proteins and Blood Glucose levels were serially recorded to assess for TPN and IVFO related toxicities.

\section{Study end points and Statistical analyses}

Five cardinal statistics were calculated for the study population:

1. Balthazar grade on CT with the highest PPV for progression to SAP in the control group at Day 0. $\left(\mathrm{B}_{\mathrm{c}}\right)$. The PPV for various Balthazar grades on CT scan on day 0 for prediction of progression from PSAP to SAP were calculated and the grade with highest PPV was determined.
2. Number of patients in the $\omega-3$ PUFA group with $\mathrm{B}_{\mathrm{c}} \cdot\left(\mathrm{N}_{\omega}\right)$

3. Number of patients predicted to progress to SAP among $\mathrm{N}_{\omega}$ by applying the calculated PPV of the Balthazar grade. $\left(\mathrm{N}_{\mathrm{p}}\right)$

The PPV for the Balthazar grade calculated from the control group was applied to the $\omega-3$ PUFA group and the number of patients who were predicted to progress to SAP in that group was calculated.

1. Number of patients actually observed to progress to SAP in $\mathrm{N}_{\omega}$. $\left(\mathrm{N}_{\mathrm{o}}\right)$

The actual number of patients progressing from PSAP to SAP with the said Balthazar grade on CT in the $\omega-3$ PUFA group was calculated.

2. Comparison of $\mathrm{N}_{\mathrm{p}} \mathrm{v} / \mathrm{s} \mathrm{N}_{\mathrm{o}}$.

For the said Balthazar grade, the actual number of patients of PSAP progressing to SAP $\left(\mathrm{N}_{\mathrm{o}}\right)$ was compared to the number of patients predicted to progress to SAP in this group $\left(\mathrm{N}_{\mathrm{p}}\right)$.

Qualitative data was analysed using standard two by two tables. A p-value of $\leq 0.05$ was considered as significant. All the statistical analysis were done using SPSS version 17.0 (SPSS Inc. released 2007. SPSS for Windows, Version 17.0. Chicago, SPSS Inc.) and Graphpad prism Instat version 3.0.

\section{Results}

Both the groups comprised of 40 patients of clinically predicted severe acute pancreatitis (PSAP), with onset within 24 hours of admission to the hospital.

Mean age of patients in the control group was 40.1 (13.28) years and that in the $\omega$-3 PUFA group was 40.05 (13.28) years. Both groups were comparable with respect to age $(\mathrm{p}=0.9866)$.

The control group comprised of 35 men and 5 women, and the $\omega-3$ PUFA group comprised of 33 men and 7 women. Both groups were comparable with respect to sex distribution within the groups.

In the control group, 37 patients had a Balthazar grade D and 3 had a Balthazar grade E on a CT done on the day of admission.

In the $\omega$-3 PUFA group, 36 patients had a Balthazar grade D and 4 had a Balthazar grade E on a CT done on the day of admission.

\section{$B_{c}=$ Balthazar grade on CT with the highest PPV for progression to SAP in the control group at Day 0.}

All 40 patients in the control group had a Balthazar grade of D and above. Of these 40 patients, 35 patients progressed to severe acute pancreatitis in the first three days of admission. 
The PPV for a Balthazar score of D and above for predicting progression to SAP was $87.5 \%$.

$\mathbf{N}_{\omega}=$ Number of patients in the $\omega-3$ PUFA group with $B_{c}$

All 40 patients in the $\omega-3$ PUFA group had a Balthazar grade of $\mathrm{D}$ and above.

\section{$N_{p}=$ Number of patients predicted to progress to SAP among $N_{\omega}$ by applying the calculated PPV of the Balthazar grade. \\ Based on the PPV calculated from the control group, 35 (87.5\%) patients were predicted to progress to SAP in the $\omega$-3 PUFA group.}

$\mathbf{N}_{\mathbf{0}}=$ Number of patients actually observed to progress to SAP in $\mathbf{N}_{\boldsymbol{\omega}}$.

$28(70 \%)$ patients actually progressed to SAP in the $\omega-3$ PUFA group.

Comparison of $\mathbf{N}_{\mathbf{p}} \mathbf{v} / \mathbf{s} \mathbf{N}_{\mathbf{o}}$

$\mathrm{N}_{\mathrm{p}}=35(87.5 \%)$ patients predicted to progress to SAP in $\omega-3$ PUFA group.

$\mathrm{N}_{\mathrm{o}}=28(70 \%)$ patients actually progressing to SAP in $\omega-3$ PUFA group.

This difference is statistically significant at $\mathrm{p}=0.0315$.

\section{Discussion}

The initial event in acute pancreatitis is intra- acinar activation of pancreatic zymogens which leads to a local inflammatory reaction and a subsequent exaggerated systemic inflammatory response syndrome (SIRS) [19-22]. The local inflammatory process leads to necrosis of pancreatic parenchyma, which further propagates the inflammatory response. Pancreatic necrosis and other local complications of acute pancreatitis can be detected on CT scan and can be used to predict the severity of acute pancreatitis. The development of the dynamic contrast injection technique allowed for the differentiation of viable from non- viable pancreatic parenchyma, thus establishing the entity of necrotizing pancreatitis. The severity of pancreatic necrosis is based on the percentage of pancreatic parenchyma that is observed to be nonviable [6-10]. Various scoring systems were developed to predict the severity of acute pancreatitis that took the morphological features of acute pancreatitis into account.

The two best known systems for this purpose are the Balthazar grade [6] and the CTSI [7,23]. A progressively increasing Balthazar grade is allotted to the presence of local complications of pancreatitis such as intra- or peri- pancreatic fluid collections. The CTSI takes into account the extent of necrosis of the pancreatic parenchyma and combines that information with the Balthazar grade to generate a CTSI score [7, 23]. Although, the CTSI has been shown to predict the severity of pancreatitis more accurately than the Balthazar grade, it is limited by its dependence on the development of pancreatic necrosis. The radiologic features of pancreatic necrosis are identified 4-7 days $[11,12]$ after the onset of pancreatits, by which time a critical window for early aggressive intervention is lost. Since the Balthazar score does not rely on the development of radiological features of pancreatic necrosis, it can be ascertained from a CT scan done earlier in the course of the disease and without the administration of IV contrast [6]. The Atlanta guidelines define acute pancreatitis based on any two of clinical, biochemical or radiological criteria [17]. A CT scan is now part of the investigative work- up of any patient presenting with acute abdominal pain. In patients of acute pancreatitis, this diagnostic CT scan can also be used to predict the severity of the disease on the day of admission itself, thus allowing for early aggressive intervention in select patients.

The treatment of acute pancreatitis at present is largely supportive. However, a therapeutic window for intervention with modulators of inflammation exists between the onset of clinical symptoms and peak proinflammatory cytokine expression. Polyunsaturated fatty acids (omega- 6 and omega- 3 fatty acids) are the precursors of the lipid mediators and play an important role in regulation of inflammation. Omega- 6 fatty acids (e.g. arachidonic acid) promote inflammation whereas omega-3 fatty acids (e.g. eicosapentanoic acid and docosahexanoic acid) have anti-inflammatory properties, dampening inflammation by inhibiting the formation of omega-6 fatty acids-derived proinflammatory eicosanoids (e.g. PGE2 and LTB4), and suppressing the activity of nuclear transcription factors, such as NFkB [15, 16]. Evidence exists that suggests that the production of inflammatory cytokines takes place systemically and not just locally within the pancreas. If these mediators are activated within end organs, such as the lungs and kidneys, the ability to 
attenuate their production may have similar beneficial effects within these organs as demonstrated within the pancreas [24, 25, 26]. Omega- 3 PUFAs have been shown to reduce levels of pro- inflammatory cytokines like IL-6, in several human trials [27, 28].

Our study showed the positive predictive value of the Balthazar score of D and above on day 0 in predicting SAP to be $87.5 \%$ in the control group, thus showing that a Balthazar grade of D and above on a CT scan done within 48 hours of onset of acute pancreatitis can accurately predict the progression of clinically suspected severe acute pancreatitis to severe acute pancreatitis.

This value was applied to the $\omega-3$ PUFA treated group to see if the actual number of patients with PSAP progressing to SAP was different than the predicted number. Of the patients in the $\omega-3$ PUFA group with Balthazar score of D and above, the number of patients observed to progress to SAP in the first 3 days were significantly lesser than the number predicted to progress [28 $(70 \%) \mathrm{v} / \mathrm{s} 35(87.5 \%) ; \mathrm{p}=0.0315]$, thus demonstrating the efficacy of $\omega-3$ PUFA in improving the early clinical outcomes of the patients of moderate to severe AP. Therefore, due consideration should be given to making IV Omega- 3 PUFA supplementation part of the standard management protocols for moderate to severe AP.

Ethical approval and consent: All procedures performed in this study were in accordance with the ethical standards of the institutional research ethics committee and with the 1964 Helsinki declaration and its later amendments or comparable ethical standards. Informed consent was obtained from all individual participants included in the study.

Disclosure: The authors report no conflict of interest.

Funding: Nil, Conflict of interest: None initiated. Permission from IRB: Yes

\section{References}

1. Steinberg WM, Goldstein SS, Davis ND, Shamma'a

J, Anderson K. Diagnostic assays in acute pancreatitis. A study of sensitivity and specificity. Ann Intern Med. 1985 May;102(5):576-80.

2. Keim V, Teich N, Fiedler F, Hartig W, Thiele G, Mössner J. A comparison of lipase and amylase in the diagnosis of acute pancreatitis in patients with abdominal pain. Pancreas. 1998 Jan;16(1):45-9.
3.Bhatia M, Brady M, Shokuhi S, Christmas S, Neoptolemos JP, Slavin J. Inflammatory mediators in acute pancreatitis. J Pathol. 2000 Feb;190(2):117-25.

4.Pooran N, Indaram A, Singh P, Bank S. Cytokines (IL-6, IL-8, TNF): early and reliable predictors of severe acute pancreatitis. J Clin Gastroenterol. 2003 Sep;37(3):263-6.

5. Riché, Florence C., et al. Inflammatory cytokines, C reactive protein, and procalcitonin as early predictors of necrosis infection in acute necrotizing pancreatitis. Surgery 2003;133(3):257-262.

6. Balthazar EJ, Robinson DL, Megibow AJ, Ranson JH. Acute pancreatitis: value of CT in establishing prognosis. Radiology. 1990 Feb;174(2):331-6.

7. Freeny PC. Angio-CT: diagnosis and detection of complications of acute pancreatitis. Hepatogastroenterology. 1991 Apr; 38(2):109-15.

8. Larvin, Michael, Alan G. Chalmers, and Michael J. McMahon. Dynamic contrast enhanced computed tomography: a precise technique for identifying and localising pancreatic necrosis. BMJ 300.6737 (1990): 1425-1428.

9. S Block, W Maier, R Bittner et al. Identification of pancreas necrosis in severe acute pancreatitis: imaging procedures versus clinical staging. Gut. 1986 Sep; 27 (9): 1035-1042.

10. Kivisaari, L et al. Early detection of acute fulminant pancreatitis by contrast-enhanced computed tomography. Scandinavian journal of gastroenterology 18.1 (1983): 39-41.

11. Leung $\mathrm{TK}^{1}$, Lee CM, Lin SY, Chen HC, Wang HJ, Shen LK, Chen YY. Balthazar computed tomography severity index is superior to Ranson criteria and APACHE II scoring system in predicting acute pancreatitis outcome. World J Gastroenterol. 2005 Oct 14;11(38):6049-52.

12.Gürleyik G, Emir S, Kiliçoglu G, Arman A, Saglam A. Computed tomography severity index, APACHE II score, and serum CRP concentration for predicting the severity of acute pancreatitis. JOP. 2005 Nov 10;6 (6) : 562-7. 
13.Wilson PG, Manji M, Neoptolemos JP. Acute pancreatitis as a model of sepsis. J Antimicrob Chemother. 1998 Jan; 41 Suppl A:51-63.

14.Balthazar EJ. Acute pancreatitis: assessment of severity with clinical and CT evaluation. Radiology. 2002 Jun; 223(3):603-13.

15. Weylandt KH, Nadolny A, Kahlke L et al. Reduction of inflammation and chronic tissue damage by omega-3 fatty acids in fat- 1 transgenic mice with pancreatitis. Biochim Biophys Acta. 2008 Nov;1782 (11):634-41. doi: 10.1016/j.bbadis.2008.08.011. Epub 2008 Sep 12.

16. Wang $\mathrm{X}^{1}, \mathrm{Li} \mathrm{W}, \mathrm{Li} \mathrm{N}, \mathrm{Li}$ J. $\omega-3$ fatty acidssupplemented parenteral nutrition decreases hyperinflammatory response and attenuates systemic disease sequelae in severe acute pancreatitis: a randomized and controlled study. JPEN J Parenter Enteral Nutr. 2008 May-Jun; 32(3):236-41. doi: 10. 1177/0148607108316189.

17. Bollen TL, van Santvoort HC, Besselink MG, van Leeuwen MS, Horvath KD, Freeny PC, Gooszen HG; Dutch Acute Pancreatitis Study Group. The Atlanta Classification of acute pancreatitis revisited. Br J Surg. 2008 Jan;95(1):6-21.

18. Marshall JC, Cook DJ, Christou NV, Bernard GR, Sprung CL, Sibbald WJ. Multiple organ dysfunction score: a reliable descriptor of a complex clinical outcome. Crit Care Med. 1995 Oct;23(10):1638-52.

19. Gorelick FS, Otani T. Mechanisms of intracellular zymogen activation. Baillieres Best Pract Res Clin Gastroenterol. 1999 Jul;13(2):227-40.

20. Steer ML. Early events in acute pancreatitis. Baillieres Best Pract Res Clin Gastroenterol. 1999 Jul;13(2):213-25.

21. Saluja, A. K., et al. Secretagogue-induced digestive enzyme activation and cell injury in rat pancreatic acini. American Journal of Physiology-Gastrointestinal and Liver Physiology 276.4 (1999): G835-G842.

22. Grady, T., et al. Zymogen proteolysis within the pancreatic acinar cell is associated with cellular injury. American Journal of Physiology-Gastrointestinal and Liver Physiology 275.5 (1998): G1010-G1017.

23. Balthazar EJ, Freeny PC, van Sonnenberg E. Imaging and intervention in acute pancreatitis. Radiology. 1994 Nov;193(2):297-306.

24. Norman, J., M. Franz, and J. Messina. Intrinsic production of proinflammatory cytokines by the pancreas during acute pancreatitis. Surg Forum. Vol. 19. 1994.

25. Formela, L. J., et al. Demonstration Of TumorNecrosis-Factor In Pancreatic Acinar-Cells By In-Situ Hybridization In A Model Of Acute-Pancreatitis.Gut. Vol. 35. No. 4. British Med Assoc House, Tavistock Square, London, England Wc1h 9jr: British Med Journal Publ Group, 1994.

26. Grewal HP, Kotb M, el Din AM, Ohman M, Salem A, Gaber L, Gaber AO. Induction of tumor necrosis factor in severe acute pancreatitis and its subsequent reduction after hepatic passage. Surgery. 1994 Feb; 115(2):213-21.

27. Wang, Xinying, et al. $\omega-3$ fatty acids-supplemented parenteral nutrition decreases hyperinflammatory response and attenuates systemic disease sequelae in severe acute pancreatitis: a randomized and controlled study. Journal of Parenteral and Enteral Nutrition 32.3 (2008): 236-241.

28. Pearce $\mathrm{CB}^{1}$, Sadek SA, Walters AM et al. A doubleblind, randomised, controlled trial to study the effects of an enteral feed supplemented with glutamine, arginine, and omega-3 fatty acid in predicted acute severe pancreatitis. JOP. 2006 Jul 10;7(4):361-71.

\section{How to cite this article?}

Rao S., Kunte A. Assessment of efficacy of IV $\omega-3$ PUFA supplementation in patients of predicted severe acute pancreatitis using the positive predictive value of Balthazar score within 48 hours of onset. Int J Med Res Rev 2016;4 (6):993-998.doi: 10.17511/ijmrr.2016.i06.22. 